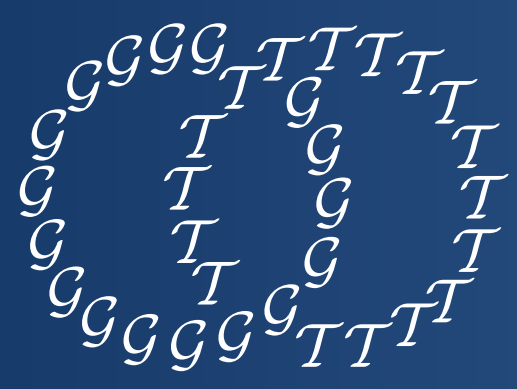

Geometry \&

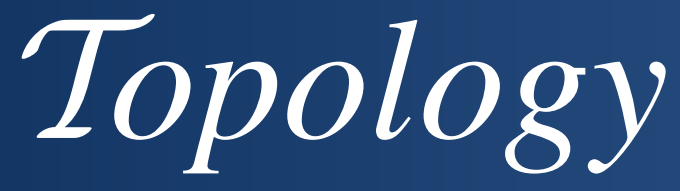

Volume 16 (2012)

On the nonexistence of certain branched covers

PEKKA PANKKA

JUAN SOUTO 


\title{
On the nonexistence of certain branched covers
}

\author{
PEKKA PANKKA \\ JUAN SOUTO
}

\begin{abstract}
We prove that while there are maps $\mathbb{T}^{4} \rightarrow \#^{3}\left(\mathbb{S}^{2} \times \mathbb{S}^{2}\right)$ of arbitrarily large degree, there is no branched cover from the 4 -torus to $\#^{3}\left(\mathbb{S}^{2} \times \mathbb{S}^{2}\right)$. More generally, we obtain that, as long as a closed manifold $N$ satisfies a suitable cohomological condition, any $\pi_{1}$-surjective branched cover $\mathbb{T}^{n} \rightarrow N$ is a homeomorphism.
\end{abstract}

This article is incorrect. Text of retraction received 14 February 2019

57M12; 30C65, 57R19

\section{Introduction}

Starting with the classical result of Alexander [1], asserting that every closed, orientable PL-manifold of dimension $n$ admits a branched cover onto the $n$-dimensional sphere $\mathbb{S}^{n}$, a series of authors such as Berstein and Edmonds [3], Edmonds [11], Hilden [15], Hirsch [16], Iori and Piergallini [18], Montesinos [19] and Piergallini [20] have proved the existence of branched covers satisfying certain conditions between certain manifolds. In this note, the starting point is a general existence theorem due to Edmonds [11]: Every $\pi_{1}$-surjective map $f: M \rightarrow N$ between closed, orientable 3 -manifolds with $\operatorname{deg}(f) \geq 3$ is homotopic to a branched cover. We show that, already in 4 dimensions, there are subtle obstructions for the existence of branched covering mappings.

Theorem 1.1 There is no branched cover from the 4-dimensional torus $\mathbb{T}^{4}$ to $\#^{3}\left(\mathbb{S}^{2} \times \mathbb{S}^{2}\right)$, the connected sum of three copies of $\mathbb{S}^{2} \times \mathbb{S}^{2}$. On the other hand, there are, a fortiori $\pi_{1}$-surjective, maps $\mathbb{T}^{4} \rightarrow \#^{3}\left(\mathbb{S}^{2} \times \mathbb{S}^{2}\right)$ of arbitrarily large degree.

Recall that a branched cover between closed PL-manifolds is a discrete and open PL-map; in the sequel we work always in the PL-category and consider only oriented manifolds and orientation preserving maps; this is no reduction of generality as the torus admits an orientation reversing involution.

One of the reasons why we find Edmonds's theorem to be rather surprising is because it asserts that, in 3 dimensions, there are many more branched covers than we would 
naively have expected. A different interpretation of the same theorem is that it is not clear how to distinguish between branched covers and maps of positive degree. When trying to rule out the existence of branched coverings between two closed manifolds $M$ and $N$, one can perhaps observe that any branched cover $f: M \rightarrow N$ induces, via pullback, an injective homomorphism

$$
f^{*}: H^{*}(N ; \mathbb{R}) \rightarrow H^{*}(M ; \mathbb{R})
$$

between the associated cohomology rings. Although this poses a huge restriction to the existence of branched covers, the same restriction applies for the existence of mere maps of nonzero degree. In fact, it is due to Duan and Wang [10] that, at least when the target $N$ is a simply connected 4-dimensional manifold, the existence of maps $M \rightarrow N$ of positive degree is equivalent to the existence of maps $H^{2}(N ; \mathbb{Z}) \rightarrow H^{2}(M ; \mathbb{Z})$ scaling the intersection form.

In the light of Theorem 1.1, the existence of branched covers is a more subtle issue which does not seem to be detected by any standard cohomological property. Cohomological considerations play a central role in the proof of Theorem 1.1, but they come into our argument in a perhaps unexpected way.

Quasiregularly elliptic manifolds: the original motivation We would like to mention that our interest to investigate the existence of branched covers from $\mathbb{T}^{4}$ onto $\#^{3}\left(\mathbb{S}^{2} \times \mathbb{S}^{2}\right)$ stems from a question of Gromov [12; 13] and Rickman [21], who asked if the manifolds $\#^{k}\left(\mathbb{S}^{2} \times \mathbb{S}^{2}\right)$ are $K$-quasiregularly elliptic for some $K$.

Recall that a continuous mapping $f: M \rightarrow N$ between oriented Riemannian $n-$ manifolds is said to be $K$-quasiregular, with (outer) distortion $K \geq 1$, if $f$ is a Sobolev mapping in $W_{\text {loc }}^{1, n}(M ; N)$ and satisfies the quasiconformality condition

$$
|D f|^{n} \leq K J_{f} \quad \text { a.e. }
$$

where $|D f|$ is the operator norm of the differential $D f$ and $J_{f}$ is the Jacobian determinant. A Riemannian manifold of dimension $n$ is $K$-quasiregularly elliptic if it admits a nonconstant $K$-quasiregular mapping from $\mathbb{R}^{n}$. We refer to Rickman [22] and BonkHeinonen [4] for a detailed discussion on quasiregular mappings and quasiregularly elliptic manifolds respectively.

Returning to the quasiregular ellipticity of manifolds $\#^{k}\left(\mathbb{S}^{2} \times \mathbb{S}^{2}\right)$, recall that Bonk and Heinonen showed in [4] that $K$-quasiregularly elliptic manifolds have quantitatively bounded cohomology. In particular, there exists a bound $k_{0}=k_{0}(K)$ depending only on $K$, so that $\#^{k}\left(\mathbb{S}^{2} \times \mathbb{S}^{2}\right)$ is not $K$-quasiregularly elliptic for $k>k_{0}$.

Without a priori bounds on the dilatation $K$, the question on the quasiregular ellipticity of manifolds $\#^{k}\left(\mathbb{S}^{2} \times \mathbb{S}^{2}\right)$ is only answered for $k=1,2$. We sketch the argument 
used in these two cases. Observing that branched covers between closed manifolds are quasiregular, it follows that every manifold $N$ for which there is a branched cover $f: \mathbb{T}^{4} \rightarrow N$ is quasiregularly elliptic. Considering $\mathbb{T}^{4}$ as the product of two $2-$ dimensional tori and taking on each factor a branched covering $\mathbb{T}^{2} \rightarrow \mathbb{S}^{2}$, one obtains a branched cover $\mathbb{T}^{4} \rightarrow \mathbb{S}^{2} \times \mathbb{S}^{2}$ and deduces that the latter manifold is quasiregularly elliptic. What is really much more surprising, is that there is also a branched cover $\mathbb{T}^{4} \rightarrow \#^{2}\left(\mathbb{S}^{2} \times \mathbb{S}^{2}\right)$; see [23] for this construction. In particular $\#^{2}\left(\mathbb{S}^{2} \times \mathbb{S}^{2}\right)$ is also quasiregularly elliptic.

The unfortunate offshoot of Theorem 1.1 is that this argument cannot be applied to $\#^{k}\left(\mathbb{S}^{2} \times \mathbb{S}^{2}\right)$ for $k=3$. Notice that for $k>3$, this observation follows directly from the injectivity of the homomorphism (1-1).

Sketch of the proof of Theorem 1.1 We will deduce the existence of maps $\mathbb{T}^{4} \rightarrow$ $\#^{3}\left(\mathbb{S}^{2} \times \mathbb{S}^{2}\right)$ with arbitrarily large degree from the work of Duan and Wang [10]. The nonexistence of branched covers in Theorem 1.1 is a special case of the following more general result:

Theorem 1.2 Let $N$ be a closed, connected, and oriented $n$-manifold, $n \geq 2$, so that $\operatorname{dim} H^{r}(N ; \mathbb{R})=\operatorname{dim} H^{r}\left(\mathbb{T}^{n} ; \mathbb{R}\right)$ for some $1 \leq r<n$. Then every branched cover $\mathbb{T}^{n} \rightarrow N$ is a cover. In particular, every $\pi_{1}$-surjective branched cover $\mathbb{T}^{n} \rightarrow N$ is a homeomorphism.

Suppose that $f: \mathbb{T}^{n} \rightarrow N$ is a branched cover as in the statement of Theorem 1.2. By a result of Berstein and Edmonds [2], there are a compact polyhedron $X$, a finite group $G$ of automorphisms of $X$ and a subgroup $H$ of $G$ such that $N=X / G$, $\mathbb{T}^{n}=X / H$ and such that the map $f$ is just the orbit map $X / H \rightarrow X / G$. Our first goal is to prove that the group $G$ acts on the $H$-invariant cohomology $H^{*}(X ; \mathbb{R})^{H}$ of $X$; this is the content of Section 3 below.

In Section 4 , we use the action of $G$ on $H^{1}(X ; \mathbb{R})^{H}$ to construct, by averaging, cocycles $\Theta_{1}, \ldots, \Theta_{n}$ which form a basis of $H^{1}(X ; \mathbb{R})^{H}$ and behave well with respect to the action of $G$ on $C^{1}(X ; \mathbb{R})$, the vector space of 1 -cochains on $X$.

These cocycles give rise to a map $X \rightarrow \mathbb{R}^{n} / \mathbb{Z}^{n}$ which semiconjugates the action of $G$ on $X$ to a certain action on $\mathbb{R}^{n} / \mathbb{Z}^{n}$. This map is defined in the same way as the classical Abel-Jacobi map and this is the way we will refer to it below. The reader can find the construction and some properties of the Abel-Jacobi map in Section 5 and Section 6. The Abel-Jacobi map and its $G$-equivariance properties are the key tools to show that the map $f: \mathbb{T}^{n} \rightarrow N$ factors as $\mathbb{T}^{n} \rightarrow N^{\prime} \rightarrow N$ where the first 
arrow is a cover and the second arrow has at most degree 2; moreover, $N^{\prime}$ is homotopy equivalent to a torus.

In Section 7 we will conclude the proof of Theorem 1.2 showing that the branched cover $N^{\prime} \rightarrow N$ is a homeomorphism. In order to do so, we have to rule out that it has degree 2. Every degree 2 branched cover is regular, meaning that there is an involution $\sigma: N^{\prime} \rightarrow N^{\prime}$ such that $N=N^{\prime} /\langle\sigma\rangle$ and that the map $N^{\prime} \rightarrow N=N^{\prime} /\langle\sigma\rangle$ is the orbit map. At this point we will already know that the involution $\sigma$ acts as -id on $H_{1}\left(N^{\prime} ; \mathbb{R}\right) \simeq \mathbb{R}^{n}$. The Lefschetz fixed-point theorem implies that the fixed-point set $\operatorname{Fix}(\sigma)$ is not empty. The final contradiction is obtained when we show, using Smith theory, that $\operatorname{Fix}(\sigma)$ is in fact empty.

Once Theorem 1.2 is proved, we discuss Theorem 1.1 in Section 8.

Acknowledgements The first author would like to thank Seppo Rickman for frequent discussions on these topics and encouragement. The second author would like to express his gratitude to the Department of Mathematics and Statistics of the University of Helsinki for its hospitality. Both authors would like to thank the referee for kind remarks and suggestions improving the manuscript. The first author has been supported by Academy of Finland project number 1126836 and by NSF grant DMS-0757732. The second author has been partially supported by NSF grant DMS-0706878, NSF Career award 0952106 and the Alfred P Sloan Foundation.

The second author also wishes to dedicate this paper to Paul el pulpo.

\section{Preliminaries}

In this section we remind the reader of a few well-known facts and definitions. Throughout this note we will work in the PL-category. We refer the reader to Hudson [17] and Rourke and Sanderson [24] for basic facts on PL-theory.

\subsection{Chains, cochains and homology}

Suppose that $X$ is a compact polyhedron, ie the geometric realization of a finite simplicial complex and let $R$ be either $\mathbb{R}$ or $\mathbb{Z}$. Denote by $C_{k}(X ; R), Z_{k}(X ; R), C^{k}(X ; R)$ and $Z^{k}(X ; R)$ the $R$-modules of singular $k$-chains, $k$-cycles, $k$-cochains and $k$ cocycles with coefficients in $R$. The associated homology and cohomology groups are denoted, as always, by $H_{k}(X ; R)$ and $H^{k}(X ; R)$. The homology (resp. cohomology) class represented by a chain (resp. cochain) $\alpha$ will be denoted by $[\alpha]$.

If $\phi: X \rightarrow Y$ is a continuous map to another polyhedron $Y$, denote by $\phi_{\#}: C_{k}(X ; R) \rightarrow$ $C_{k}(Y ; R)$ and by $\phi^{\#}: C^{k}(Y ; R) \rightarrow C^{k}(X ; R)$ the pushforward and pullback of chains 
and cochains, respectively. On homological level, we denote the pushforward and pullback by $\phi_{*}: H_{*}(X ; R) \rightarrow H_{*}(Y ; R)$ and $\phi^{*}: H^{*}(Y ; R) \rightarrow H^{*}(X ; R)$ respectively. Finally, denote by

$$
\langle\cdot, \cdot\rangle: C^{k}(X ; R) \times C_{k}(X ; R) \rightarrow R
$$

the canonical evaluation form. Notice that, with the same notation as above, we have for $\omega \in C^{k}(Y ; R)$ and $c \in C_{k}(X ; R)$

$$
\left\langle\phi^{\#}(\omega), c\right\rangle=\left\langle\omega, \phi_{\#}(c)\right\rangle .
$$

The induced bilinear form on $H^{k}(X ; R) \times H_{k}(X ; \mathbb{R})$ is also denoted by $\langle\cdot, \cdot\rangle$; again (2-1) is satisfied

$$
\left\langle\phi^{*}([\omega]),[c]\right\rangle=\left\langle[\omega], \phi_{*}([c])\right\rangle .
$$

Recall that if $c$ and $\omega$ are a chain and a cochain representing the homology and cohomology classes $[c]$ and $[\omega]$ we have $\langle\omega, c\rangle=\langle[\omega],[c]\rangle$.

Continuing with the same notation, suppose that $G$ is a finite group acting on the polyhedron $X$. We denote by $X / G$ the quotient space and by

$$
\pi_{G}: X \rightarrow X / G
$$

the orbit map. The $G$-invariant real cohomology

$$
H^{*}(X ; \mathbb{R})^{G}=\left\{\omega \in H^{*}(X ; \mathbb{R}) \mid g^{*}(\omega)=\omega \text { for all } g \in G\right\}
$$

of $X$ is isomorphic to the real cohomology $H^{*}(X / G ; \mathbb{R})$ of the orbit space $X / G$. Given that this fact will be used over and over again, we state it as a proposition:

Proposition 2.1 Let $X$ be a polyhedron and $G$ a finite group acting on $X$. The pullback of the orbit map $\pi_{G}: X \rightarrow X / G$ induces an isomorphism between $H^{*}(X ; \mathbb{R})^{G}$ and $H^{*}(X / G ; \mathbb{R})$.

Proposition 2.1 is a consequence of the natural transfer homomorphism $H_{*}(X / G ; \mathbb{R}) \rightarrow$ $H_{*}(X ; \mathbb{R})$; see eg Bredon [6, III.2]. Below we will need the following closely related fact, whose proof we leave to the reader:

Lemma 2.2 Let $X$ be a polyhedron, $G$ a finite group acting on $X$ and suppose that $c$ is a $k$-chain whose pushforward $\left(\pi_{G}\right)_{\#}(c)$ under the orbit map $\pi_{G}: X \rightarrow X / G$ is a $k$-cycle in $X / G$. Then $\sum_{g \in G} g_{\#}(c)$ is a $k$-cycle in $X$.

Moreover, if $[\omega]$ is a $k$-cohomology class in $X / G$ then

$$
\left\langle\pi_{G}^{*}([\omega]),\left[\sum_{g \in G} g_{\#}(c)\right]\right\rangle=|G|\left\langle[\omega],\left[\left(\pi_{G}\right)_{\#}(c)\right]\right\rangle .
$$


We refer the reader to $[6 ; 7 ; 14]$ for basic facts of algebraic topology.

\subsection{Branched covers}

A branched cover between oriented PL-manifolds is an orientation preserving discrete and open PL-map. Given any such branched cover $f: M \rightarrow N$ we say that $x \in M$ is a singular point of $f$ if $f$ is not a local homeomorphism in a neighborhood of $x$. The image $f(x)$ of a singular point is a branching point of $f$. We denote by $S_{f} \subset M$ and $B_{f}=f\left(S_{f}\right) \subset N$ the sets of singular points and branch points of $f$ respectively. We also denote by $Z_{f}=f^{-1}\left(B_{f}\right)$ the preimage of the set of branching points of $f$; notice that $S_{f} \subset Z_{f}$.

Before moving on, observe that since $f$ is PL, discrete and open, the sets $S_{f}, B_{f}$ and $Z_{f}$ are polyhedra of codimension at least 2. Remark also that the restriction of $f$ to $M \backslash Z_{f}$ is a covering map onto $N \backslash B_{f}$.

Remark It is worth noticing that the above statement does not require the mapping to be PL. Indeed, given a discrete and open mapping between $n$-manifolds the singular set has topological codimension at least 2 by a result of Černavskiǔ [8] and Väisälä [25]. Moreover, $B_{f}$ and $S_{f}$ have the same (topological) dimension.

A branched cover $f: M \rightarrow N$ is said to be regular if there is a group $G$ of PLautomorphisms of $M$ such that there is an identification of $N$ with $M / G$ in such a way that the map $f$ becomes the orbit map $\pi_{G}: M \rightarrow M / G=N$. The following result, due to Berstein and Edmonds [2, Proposition 2.2], asserts that every branched cover is a, nonregular, orbit map:

Proposition 2.3 Given a branched cover $f: M \rightarrow N$ between closed orientable manifolds of dimension $n$, there exists a connected polyhedron $X_{f}$ of dimension $n$, a group $G$ of automorphisms of $X_{f}$, a subgroup $H$ of $G$ and identifications $M=X_{f} / H$ and $N=X_{f} / G$ so that the following diagram commutes:

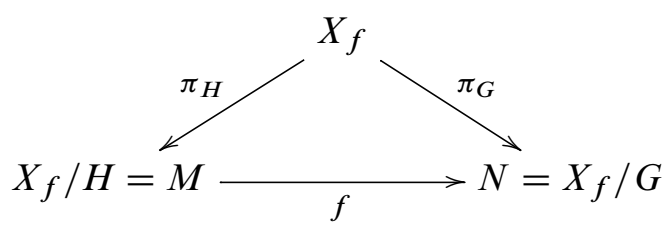

Moreover, $H^{n}\left(X_{f}, \mathbb{R}\right) \simeq \mathbb{R}$ and $G$ acts trivially on $H^{n}\left(X_{f}, \mathbb{R}\right)$. 
We observe that in general the polyhedron $X_{f}$ is not a manifold. However, the complement of $\pi_{G}^{-1}\left(B_{f}\right)=\pi_{H}^{-1}\left(Z_{f}\right)$ in $X_{f}$ is a manifold and the restriction of $\pi_{G}$ and $\pi_{H}$ to that set is a covering map. Just to clarify terminology, we would like to mention that under a cover or covering map we understand, as usual, a branched cover whose singular set is empty.

Before going any further we state, with the same notation as in Proposition 2.3, the following simple consequence of the fact that $H^{n}\left(X_{f} ; \mathbb{R}\right) \simeq \mathbb{R}$ and that $G$ acts trivially on $H^{n}\left(X_{f} ; \mathbb{R}\right)$. If $K$ is namely a subgroup of $G$ then $H^{n}\left(X_{f} / K ; \mathbb{R}\right) \simeq \mathbb{R}$ by Proposition 2.1. Hence, if $V_{1}, V_{2}$ are oriented $n$-manifolds then any two continuous maps

$$
f_{1}: V_{1} \rightarrow X_{f} / K, \quad f_{2}: X_{f} / K \rightarrow V_{2}
$$

have a well-defined degree $\operatorname{deg}\left(f_{i}\right)$ which satisfies the usual rule

$$
\operatorname{deg}\left(f_{2} \circ f_{2}\right)=\operatorname{deg}\left(f_{2}\right) \operatorname{deg}\left(f_{1}\right) .
$$

This fact will be of some importance below.

\subsection{Branched covers of degree 1 or 2}

Below, we will show that any branched cover $f: \mathbb{T}^{n} \rightarrow N$ as in the statement of Theorem 1.2 is the composition of a covering map and a branched cover of degree one or two. It is well-known that such branched covers are very particular:

\section{Proposition 2.4}

(1) A branched cover of degree 1 is a homeomorphism.

(2) Let $f: M \rightarrow N$ be a degree 2 branched cover between closed, connected, orientable PL-manifolds. Then there is an orientation preserving involution $\sigma: M \rightarrow M$ such that $N=M /\langle\sigma\rangle$ and such that the map $f$ is just the natural orbit map $M \rightarrow M /\langle\sigma\rangle=N$.

In the statement of Proposition 2.4, as well as in the future, we denote by $\langle\sigma\rangle$ the group generated by $\sigma$.

We conclude this section with the following observations on the fixed point sets of involutions on $n$-manifolds that have the homotopy type of an $n$-torus.

Proposition 2.5 Let $M$ be a closed orientable $n$-manifold with nontrivial orientationpreserving involution $\sigma$ such that $M /\langle\sigma\rangle$ is an $n$-manifold. Then each component of the fixed point set of $\sigma$ is a $\mathbb{Z}_{2}$-cohomology $(n-2)$-manifold. In particular, if $n \geq 3$ then the components of $\operatorname{Fix}(\sigma)$ are not $\mathbb{Z}_{2}$-acyclic. 
By classical Smith theory, the fixed point set of a $\mathbb{Z}_{p}$-action, for $p$ prime, on an orientable manifold is a $\mathbb{Z}_{p}$-cohomology $r$-manifold for some $r<n$; see eg [5, Theorem 7.4].

Recall that a $\mathbb{Z}_{p}$-cohomology $r$-manifold, for $p$ prime, is a topological space satisfying a few properties, the most important of which is that it admits a cover $\mathcal{B}$ such that the one-point compactification of every element in $\mathcal{B}$ has the same $\mathbb{Z}_{p}$-cohomology relative to the point at infinity, as the $r$-dimensional sphere $\mathbb{S}^{r}$ relative to one of its points. See [5, Definition 1.1] for details and notice that it follows directly from the definition that a connected polyhedron which is a $\mathbb{Z}_{p}$-cohomology 0 -manifold is a singleton.

Proof of Proposition 2.5 By the comment above, it suffices to show that $r=n-2$. Let $\pi: M \rightarrow M /\langle\sigma\rangle$ be the quotient map.

Let $\Sigma$ be a component of $\operatorname{Fix}(\sigma)$ and $A$ a top dimensional simplex in $\Sigma$. Then $A$ is an $r$-simplex. Let $x \in A$ be an interior point. By passing to a subdivision on $M$ and $M /\langle\sigma\rangle$ if necessary, we may assume that the link $\operatorname{Lk}(\pi(x), M /\langle\sigma\rangle)$ is an $(n-1)-$ sphere and that $\pi \mid \operatorname{Lk}(x, M): \operatorname{Lk}(x, M) \rightarrow \operatorname{Lk}(\pi(x), M /\langle\sigma\rangle)$ is a simplicial map.

Let $S_{1}=A \cap \operatorname{Lk}(x, M)$ and $S_{2}=\operatorname{Lk}(A, M)$. Then $S_{1}$ and $S_{2}$ are $r-$ and $(n-r-1)-$ spheres in $\operatorname{Lk}(x, M)$, respectively. Moreover, $\sigma$ acts trivially on $S_{1}$ and freely on $S_{2}$ by top dimensionality of $A$. Thus $\pi\left(S_{2}\right)$ has the homotopy type of $\mathbb{R} P^{n-r-1}$.

Let $\Sigma=\operatorname{Lk}(\pi(x), M /\langle\sigma\rangle)$. Since $\Sigma$ is a join of $\pi\left(S_{1}\right)$ and $\pi\left(S_{2}\right)$ and the pair $\left(\Sigma, \pi\left(S_{1}\right)\right)$ is the standard sphere pair $\left(\mathbb{S}^{n-1}, \mathbb{S}^{r-1}\right)$, we have that $\Sigma \backslash \pi\left(S_{1}\right)$ has the homotopy type of both $\mathbb{S}^{n-r-1}$ and $\mathbb{R} P^{n-r-1}$. The claim now follows.

Proposition 2.6 Let $M$ be an $n$-manifold with the homotopy type of the $n$-torus and let $\sigma$ be an involution of $M$ that acts by multiplication by $(-1)$ on $H^{1}(M, \mathbb{Z})$. Then the fixed point set $\operatorname{Fix}(\sigma)$ consists of $2^{n}$ points.

Proof Since $M$ is homotopy equivalent to an $n$-torus, we may identify $\pi_{1}(M)=$ $H_{1}(M ; \mathbb{Z})=\mathbb{Z}^{n}$ and $H^{*}(M ; R)=H^{*}\left(\mathbb{T}^{n} ; R\right)$, where $R$ is either $\mathbb{Z}$ or $\mathbb{R}$.

Since $\sigma$ acts by $(-1)$ to $H^{1}(M, \mathbb{Z})$ and $H^{*}(M, \mathbb{Z})$ is isomorphic to the ring $\bigwedge^{*} \mathbb{Z}^{n}$, we know $\sigma$ acts on $H^{s}(M ; \mathbb{Z})$ by multiplication by $(-1)^{s}$. In particular, $\sigma: M \rightarrow M$ has Lefschetz number $L(\sigma)=2^{n}$. It follows from the Lefschetz fixed point theorem that

$\operatorname{Fix}(\sigma) \neq \varnothing$ 
We show now that components of $\operatorname{Fix}(\sigma)$ are points. The claim then follows from the Lefschetz-Hopf theorem.

Let $N=M /\langle\sigma\rangle$. We observe that $H^{1}(N ; \mathbb{R})=0$. Indeed,

$$
H^{1}(N ; \mathbb{R})=H^{1}(M /\langle\sigma\rangle ; \mathbb{R})=H^{1}(M ; \mathbb{R})^{\langle\sigma\rangle}=0 .
$$

We prove first that the inclusion of $\operatorname{Fix}(\sigma)$ into $M$ is trivial on homology.

Claim 1 The map $H_{1}(\operatorname{Fix}(\sigma) ; \mathbb{Z}) \rightarrow H_{1}(M ; \mathbb{Z})$ is trivial.

Seeking a contradiction suppose that $[\alpha] \in H_{1}(\operatorname{Fix}(\sigma) ; \mathbb{Z})$ is not trivial in $H_{1}(M ; \mathbb{Z})$ and let $[\beta] \in H^{n-1}(M ; \mathbb{R})$ be the unique cohomology class with

$$
\langle[\eta],[\alpha]\rangle=[\eta] \wedge[\beta]
$$

for all $[\eta] \in H^{1}(M ; \mathbb{R})$. Since $[\alpha]$ is fixed by $\sigma$ we deduce that $[\beta]$ is also fixed by $\sigma$. This implies that

$$
H^{n-1}(M /\langle\sigma\rangle ; \mathbb{R})=H^{n-1}(M ; \mathbb{R})^{\langle\sigma\rangle} \neq 0,
$$

contradicting (2-4). This completes the proof of Claim 1.

Claim 2 Every connected component $\Sigma$ of $\operatorname{Fix}(\sigma)$ lifts homeomorphically to the universal cover $\widetilde{M}$ of $M$.

Since the homomorphism $\pi_{1}(M) \rightarrow H_{1}(M ; \mathbb{R})$ is injective, given a connected component $\Sigma$ of $\operatorname{Fix}(\sigma)$ we have the following commutative diagram:

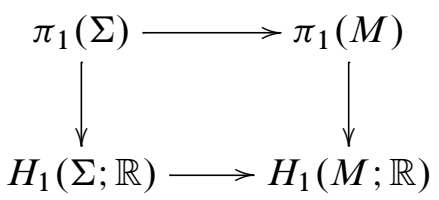

As we just observed the right vertical arrow is injective. On the other hand, the lower horizontal arrow is trivial by Claim 1. This implies that the homomorphism $\pi_{1}(\Sigma) \rightarrow \pi_{1}(M)$ is trivial as well. In other words, $\Sigma$ lifts homeomorphically to the universal cover $\widetilde{M}$ of $M$. Thus Claim 2 holds.

Let now $\Sigma \subset \operatorname{Fix}(\sigma)$ be a connected component and lift it to $\widetilde{\Sigma} \subset \widetilde{M}$. Since $M$ is homotopy equivalent to $\mathbb{T}^{n}, \widetilde{M}$ is homotopy equivalent to $\mathbb{R}^{n}$ and hence contractible. Choosing a point $\tilde{x} \in \tilde{\Sigma}$ there is a unique lift $\tilde{\sigma}$ of $\sigma$ to $\widetilde{M}$ with $\tilde{\sigma}(\tilde{x})=\tilde{x}$. Observe that $\tilde{\sigma}$ is an involution and that

$$
\widetilde{\Sigma} \subset \operatorname{Fix}(\widetilde{\sigma}) .
$$


The involution $\widetilde{\sigma}$ is obviously a transformation of the contractible space $\widetilde{M}$ of prime period 2. It is a standard consequence of Smith theory [6, III. Theorem 5.2] that Fix $(\widetilde{\sigma})$ is $\mathbb{Z}_{2}$-acyclic and hence connected. Since $\operatorname{Fix}(\widetilde{\sigma})$ is $\mathbb{Z}_{2}$-cohomology manifold, we observe that $\widetilde{\Sigma}=\operatorname{Fix}(\widetilde{\sigma})$ is a point by $\mathbb{Z}_{2}$-acyclicity and connectedness. Thus $\Sigma$ is a point. This concludes the proof of Proposition 2.6.

We combine these two propositions to the following corollary.

Corollary 2.7 Let $M$ be an $n$-manifold with the homotopy type of the $n$-torus. For $n \geq 3$ there is no involution $\sigma$ on $M$ that acts by multiplication by $(-1)$ on $H^{1}(M ; \mathbb{Z})$ and $M /\langle\sigma\rangle$ is a manifold.

Remark An equivalent formulation for Corollary 2.7 is that if $M$ and $N$ are PLmanifolds, with $M$ homotopy equivalent to the $n$-dimensional torus with $n \geq 3$ and with $H_{1}(N ; \mathbb{R})=0$, then there is no 2 -fold branched cover $M \rightarrow N$. In the topological category, this result is due to Connolly, Davis and Kahn [9].

At this point we fix some notation that will be used throughout the whole paper:

Notation 2.8 Suppose that $f: \mathbb{T}^{n} \rightarrow N$ is a branched cover as in the statement of Theorem 1.2; in other words, we may fix $1 \leq r<n$ so that $\operatorname{dim} H^{r}(N ; \mathbb{R})=$ $\operatorname{dim} H^{r}\left(\mathbb{T}^{n} ; \mathbb{R}\right)$. Let also $X=X_{f}$ be the polyhedron provided by Proposition 2.3 and $H \subset G$ the groups of automorphisms of $X$ with $\mathbb{T}^{n}=X / H$ and $N=X / G$. Also, let

$$
\pi=\pi_{H}: X \rightarrow X / H=\mathbb{T}^{n}
$$

be the orbit map onto $X / H$.

\section{The key observation}

The key tool in the proof of Theorem 1.2 is the analysis of the action of $G$ on the singular (real) cohomology ring $H^{*}(X ; \mathbb{R})$ of $X$.

Recall that if $\Gamma \subset G$ denotes a subgroup of $G$, then $H^{*}(X ; \mathbb{R})^{\Gamma}$ is the $\Gamma$-invariant cohomology of $X$ and that the orbit map $X \rightarrow X / \Gamma$ induces, via the pullback, an isomorphism between $H^{*}(X / \Gamma ; \mathbb{R})$ and $H^{*}(X ; \mathbb{R})^{\Gamma}$.

Since $H$ is a subgroup of $G$, it is immediate that $H^{*}(X ; \mathbb{R})^{G}$ is $H$-invariant. The main result of this section is a partial converse. Our notation is as in Notation 2.8. 
Proposition 3.1 The subspace $H^{1}(X ; \mathbb{R})^{H}$ is $G$-invariant. Moreover, for any $g \in G$ we have that the restriction of $g^{*}$ to $H^{1}(X ; \mathbb{R})^{H}$ is equal to $\pm \mathrm{id}$.

We begin with two observations.

Lemma 3.2 Elements of $G$ act trivially on $H^{r}(X ; \mathbb{R})^{H}$.

Proof Observe that

$$
\operatorname{dim} H^{r}(X ; \mathbb{R})^{G}=\operatorname{dim} H^{r}(X / G ; \mathbb{R})=\operatorname{dim} H^{r}(N ; \mathbb{R})
$$

and, similarly,

$$
\operatorname{dim} H^{r}(X ; \mathbb{R})^{H}=\operatorname{dim} H^{r}(X / H ; \mathbb{R})=\operatorname{dim} H^{r}\left(\mathbb{T}^{n} ; \mathbb{R}\right) .
$$

Since $\operatorname{dim} H^{r}(N ; \mathbb{R})=\operatorname{dim} H^{r}\left(\mathbb{T}^{n} ; \mathbb{R}\right)$ and $H^{r}(X ; \mathbb{R})^{G} \subset H^{r}(X ; \mathbb{R})^{H}$, it follows that $H^{r}(X ; \mathbb{R})^{G}=H^{r}(X ; \mathbb{R})^{H}$. Thus $G$ acts trivially on $H^{r}(X ; \mathbb{R})^{H}$ by definition.

Lemma 3.3 Let $r \geq 1, V$ be a vector space, $W$ a subspace of $V$ with $\operatorname{dim} W \geq r+1$, and $\varphi: V \rightarrow V$ a linear mapping so that $\bigwedge^{r} \varphi=\mathrm{id}: \bigwedge^{r} W \rightarrow \bigwedge^{r} W$. Then $W$ is $\varphi$-invariant and $\varphi \mid W= \pm$ id.

Proof Since $\bigwedge^{r} \varphi=\mathrm{id}$ on $\bigwedge^{r} W$, all $r$-dimensional subspaces of $W$ are invariant under $\varphi$; hence $W$ is $\varphi$-invariant. Let $v \in W, v \neq 0$, and let $U_{1}, \ldots, U_{s}$ be $r-$ dimensional subspaces of $W$ whose intersection $L=\bigcap_{i} U_{i}$ is the line containing $v$. Since subspaces $U_{i}, i=1, \ldots, s$, are $\varphi$-invariant, the line $L$ is also invariant. It follows that the restriction of $\varphi$ to $W$ fixes every line in $L$ and hence is a homothety of ratio $\lambda \in \mathbb{R}: \varphi(v)=\lambda v$ for all $v \in W$.

We claim that $\lambda= \pm 1$. In order to see that this is the case let $v_{1}, \ldots, v_{r} \in W$ with $v_{1} \wedge \cdots \wedge v_{r} \neq 0$ and compute

$$
\begin{aligned}
v_{1} \wedge \cdots \wedge v_{r} & =\left(\wedge^{r} \varphi\right)\left(v_{1} \wedge \cdots \wedge v_{r}\right)=\varphi\left(v_{1}\right) \wedge \cdots \wedge \varphi\left(v_{r}\right) \\
& =\left(\lambda v_{1}\right) \wedge \cdots \wedge\left(\lambda v_{r}\right)=\lambda^{r}\left(v_{1} \wedge \cdots \wedge v_{r}\right) .
\end{aligned}
$$

The claim follows.

Proof of Proposition 3.1 We are going to apply Lemma 3.3 to $V=H^{1}(X ; \mathbb{R}), W=$ $H^{1}(X ; \mathbb{R})^{H}$ and $\varphi=g^{*}$ for $g \in G$. Notice that $\operatorname{dim} H^{1}(X ; \mathbb{R})^{H}=\operatorname{dim} H^{1}\left(\mathbb{T}^{n} ; \mathbb{R}\right)=$ $n \geq r+1$. 
Consider the commutative diagram

$$
\begin{gathered}
\bigwedge^{r}\left(H^{1}(X / H ; \mathbb{R})\right) \stackrel{\wedge}{\longrightarrow} H^{r}(X / H ; \mathbb{R}) \\
\wedge^{r} \pi^{*} \downarrow \\
\bigwedge^{r}\left(H^{1}(X ; \mathbb{R})^{H}\right) \longrightarrow \pi^{*} \downarrow
\end{gathered}
$$

where the vertical arrows are the pullback of the orbit map $\pi: X \rightarrow X / H$, while the horizontal maps are the cup product in cohomology. By Proposition 2.1, the two vertical arrows are isomorphisms. On the other hand, the upper horizontal arrow is also an isomorphism because $X / H=\mathbb{T}^{n}$ is an $n$-dimensional torus. This proves that the homomorphism

$$
\bigwedge^{r}\left(H_{1}(X ; \mathbb{R})^{H}\right) \rightarrow H^{r}(X ; \mathbb{R})^{H}
$$

is an isomorphism.

Lemma 3.2 says $g^{*}$ acts trivially on $H^{r}(X ; \mathbb{R})^{H}$ and hence on $\bigwedge^{r}\left(H_{1}(X ; \mathbb{R})^{H}\right)$. In other words, Lemma 3.3 applies and the claim follows.

Let $G \rightarrow \operatorname{Hom}\left(H^{1}(X ; \mathbb{R})^{H}, H^{1}(X ; \mathbb{R})^{H}\right)$ be the map $g \mapsto g^{*}$. By Proposition 3.1, the image of this homomorphism is $\{ \pm \mathrm{id}\}$. Identifying $\{ \pm \mathrm{id}\}$ with the multiplicative group $\{ \pm 1\}$ of two elements, we obtain a homomorphism

$$
\delta: G \rightarrow\{ \pm 1\} .
$$

Notice that by definition $H \subset \operatorname{Ker}(\delta)$.

With the same notation as above we can now show that $H^{*}(X ; \mathbb{R})^{H}$ is in fact $G-$ invariant and describe the action

$$
G \curvearrowright H^{*}(X ; \mathbb{R})^{H} .
$$

Corollary 3.4 For $s=0,1, \ldots, n$ and $[\omega] \in H^{s}(X ; \mathbb{R})^{H}$ we have

$$
g^{*}[\omega]=\delta(g)^{s}[\omega]
$$

for all $g \in G$.

Proof Using the same argument as in the proof of Proposition 3.1, more concretely using diagram (3-1) and the discussion following that diagram, we deduce that the standard homomorphism

$$
\bigwedge^{s}\left(H_{1}(X ; \mathbb{R})^{H}\right) \rightarrow H^{s}(X ; \mathbb{R})^{H}
$$


is an isomorphism. By Proposition 3.1, and by definition of $\delta(\cdot)$, we have that $g$ acts on $H^{1}(X ; \mathbb{R})^{H}$ by multiplication by $\delta(g)$. Hence, $g$ acts on $H^{s}(X ; \mathbb{R})^{H}$ by multiplication by $\delta(g)^{S}$, as we claimed.

From here we obtain the first nontrivial restrictions on any manifold $N$ as in Notation 2.8.

Corollary 3.5 Using Notation 2.8 the following holds:

(1) The pullback $f^{*}: H^{2 *}(N ; \mathbb{R}) \rightarrow H^{2 *}\left(\mathbb{T}^{n} ; \mathbb{R}\right)$ is an isomorphism.

(2) If $n$ or $r$ is odd, then $f^{*}: H^{*}(N ; \mathbb{R}) \rightarrow H^{*}\left(\mathbb{T}^{n} ; \mathbb{R}\right)$ is an isomorphism.

Proof Since $H^{s}(N ; \mathbb{R})=H^{s}(X ; \mathbb{R})^{G} \subset H^{s}(X ; \mathbb{R})^{H}$ we deduce from Corollary 3.4 that

$$
H^{S}(N ; \mathbb{R})=\left(H^{s}(X ; \mathbb{R})^{H}\right)^{G}
$$

for $s$ even. Also by Corollary 3.4 we have that the action $G \curvearrowright H^{s}(X ; \mathbb{R})^{H}$ is trivial for even $s$; hence

$$
H^{s}(N ; \mathbb{R})=\left(H^{s}(X ; \mathbb{R})^{H}\right)^{G}=H^{s}(X ; \mathbb{R})^{H}=H^{s}\left(\mathbb{T}^{n} ; \mathbb{R}\right)
$$

for even $s$. This proves (1).

Suppose now, for the sake of concreteness, that $n$ is odd. Notice that on the one hand $g^{*}$ is trivial on $H^{n}(X ; \mathbb{R})$ by Proposition 2.3 while on the other hand it acts by multiplication by $\delta(g)^{n}$ by Corollary 3.4. Hence $\delta(g)=1$ for all $g \in G$. Observe that the same argument applies if $r$ is odd. Once we know that $\delta(g)=1$ for all $g \in G$, the second claim follows as (1).

\section{Averaging}

In this section we construct $n$ cocycles in $C^{1}(X ; \mathbb{R})$ which represent a basis of $H^{1}(X ; \mathbb{R})^{H}$ and have some highly desirable properties; notation is as in Notation 2.8. To begin, choose bases $\left[c_{1}\right], \ldots,\left[c_{n}\right]$ of $H_{1}\left(\mathbb{T}^{n} ; \mathbb{Z}\right)$ and $\left[\theta_{1}\right], \ldots,\left[\theta_{n}\right]$ of $H^{1}\left(\mathbb{T}^{n} ; \mathbb{R}\right)$ with

$$
\left\langle\left[\theta_{i}\right],\left[c_{j}\right]\right\rangle=\delta_{i j},
$$

where $\delta_{i j}$ is the Kronecker symbol.

Proposition 4.1 There are $1-$ cocycles $\Theta_{1}, \ldots, \Theta_{n} \in C^{1}(X ; \mathbb{R})$ with the following properties:

(1) $\pi^{*}\left(\left[\theta_{i}\right]\right)=\left[\Theta_{i}\right]$ for $i=1, \ldots, n$. 
(2) $g^{\#}\left(\Theta_{i}\right)=\delta(g) \Theta_{i}$ for any $g \in G$ and $i=1, \ldots, n$. Here $\delta(\cdot)$ is the homomorphism (3-2).

(3) $\left\langle\Theta_{i}, \widetilde{c}_{j}\right\rangle=\delta_{i j}$ for any $1-$ chain $\tilde{c}_{j} \in C_{1}(X ; \mathbb{R})$ in $X$ such that $\pi_{\#}\left(\tilde{c}_{j}\right)$ is 1 -cycle representing $\left[c_{j}\right]$.

(4) $\left\langle\Theta_{i}, c\right\rangle \in \mathbb{Z}$ for every integral 1 -chain $c \in C_{1}(X ; \mathbb{Z})$ whose pushforward $\pi_{\#}(c)$ is a $1-$ cycle in $X / H=\mathbb{T}^{n}$.

Proof We begin choosing 1-cocycles $\theta_{1}, \ldots, \theta_{n} \in C^{1}\left(\mathbb{T}^{n} ; \mathbb{R}\right)$ representing the cohomology classes $\left[\theta_{1}\right], \ldots,\left[\theta_{n}\right]$ and define $\Theta_{i}$ as a twisted $G$-average of the pullback $\pi^{\#}\left(\theta_{i}\right)$ :

$$
\Theta_{i}=\frac{1}{|G|} \sum_{g \in G} \delta(g) g^{\#}\left(\pi^{\#}\left(\theta_{i}\right)\right) .
$$

Observe that $\Theta_{i}$ is a cocycle because it is a weighted sum of the cocycles $g^{\#}\left(\pi^{\#}\left(\theta_{i}\right)\right)$. We claim that the cocycles $\Theta_{1}, \ldots, \Theta_{n}$ fulfill the claims of Proposition 4.1.

To show (1), notice that, since $\pi^{\#}\left(\theta_{i}\right)$ represents the cohomology class $\pi^{*}\left(\left[\theta_{i}\right]\right) \in$ $\pi^{*}\left(H^{1}(X / H ; \mathbb{R})\right)=H^{1}(X ; \mathbb{R})^{H}$, we have by Proposition 3.1 that

$$
\left[g^{\#}\left(\pi^{\#}\left(\theta_{i}\right)\right)\right]=g^{*}\left[\pi^{\#}\left(\theta_{i}\right)\right]=\delta(g) \pi^{*}\left(\left[\theta_{i}\right]\right) .
$$

Since $\delta(g)^{2}=1$ for all $g \in G$ we have

$$
\left[\Theta_{i}\right]=\left[\frac{1}{|G|} \sum_{g \in G} \delta(g) g^{\#}\left(\pi^{\#}\left(\theta_{i}\right)\right)\right]=\frac{1}{|G|} \sum_{g \in G} \delta(g)^{2} \pi^{*}\left(\left[\theta_{i}\right]\right)=\pi^{*}\left[\theta_{i}\right],
$$

as claimed.

The validity of (2) follows from a simple computation and the fact that $\delta$ is a homomorphism.

To show (3) observe that the homomorphism $\delta$ from (3-2) induces a well-defined map

$$
\delta: G / H \rightarrow\{ \pm 1\}
$$

because $H \subset \operatorname{Ker}(\delta)$. From now on we choose a representative $g$ for every class $g H \in G / H$ and hence identify $G / H$ with a subset of $G$. We start rewriting $\left\langle\Theta_{i}, \tilde{c}_{j}\right\rangle$ :

$$
\begin{aligned}
\left\langle\Theta_{i}, \tilde{c}_{j}\right\rangle & =\frac{1}{|G|}\left\langle\sum_{g \in G} \delta(g) g^{\#}\left(\pi^{\#}\left(\theta_{i}\right)\right), \tilde{c}_{j}\right\rangle \\
& =\frac{1}{|G|}\left\langle\pi^{\#}\left(\theta_{i}\right), \sum_{g \in G} \delta(g) g_{\#}\left(\tilde{c}_{j}\right)\right\rangle
\end{aligned}
$$




$$
\begin{aligned}
& =\frac{1}{|G|}\left\langle\pi^{\#}\left(\theta_{i}\right), \sum_{g \in G / H} \sum_{h \in H} \delta(g h)(g h)_{\#}\left(\tilde{c}_{j}\right)\right\rangle \\
& =\frac{1}{|G|}\left\langle\pi^{\#}\left(\theta_{i}\right), \sum_{g \in G / H} \sum_{h \in H} \delta(g) g_{\#}\left(h_{\#}\left(\tilde{c}_{j}\right)\right)\right\rangle \\
& =\frac{1}{|G|}\left\langle\pi^{\#}\left(\theta_{i}\right), \sum_{g \in G / H} \delta(g) g_{\#}\left(\sum_{h \in H} h_{\#}\left(\widetilde{c}_{j}\right)\right)\right\rangle \\
& =\frac{1}{|G|}\left\langle\sum_{g \in G / H} \delta(g) g^{\#}\left(\pi^{\#}\left(\theta_{i}\right)\right), \sum_{h \in H} h_{\#}\left(\widetilde{c}_{j}\right)\right\rangle .
\end{aligned}
$$

Notice that, by Lemma $2.2, \sum_{h \in H} h_{\#}\left(\widetilde{c}_{j}\right)$ is a 1-cycle satisfying

$$
\pi_{*}\left(\left[\sum_{h \in H} h_{\#}\left(\tilde{c}_{j}\right)\right]\right)=|H|\left[c_{j}\right] .
$$

Observing that in the last equation in (4-1) we are evaluating a 1-cocycle and a 1-cycle we obtain the same result if we evaluate the corresponding cohomology and homology classes:

$$
\begin{aligned}
\left\langle\Theta_{i}, \tilde{c}_{j}\right\rangle & =\frac{1}{|G|}\left\langle\sum_{g \in G / H} \delta(g) g^{\#}\left(\pi^{\#}\left(\theta_{i}\right)\right), \sum_{h \in H} h_{\#}\left(\tilde{c}_{j}\right)\right\rangle \\
& =\frac{1}{|G|}\left\langle\left[\sum_{g \in G / H} \delta(g) g^{\#}\left(\pi^{\#}\left(\theta_{i}\right)\right)\right],\left[\sum_{h \in H} h_{\#}\left(\tilde{c}_{j}\right)\right]\right\rangle \\
& =\frac{1}{|G|}\left\langle\sum_{g \in G / H} \delta(g) g^{*}\left(\pi^{*}\left(\left[\theta_{i}\right]\right)\right),\left[\sum_{h \in H} h_{\#}\left(\tilde{c}_{j}\right)\right]\right\rangle .
\end{aligned}
$$

By Proposition 3.1, and $\delta(g)^{2}=1$, we have

$$
\delta(g) g^{*}\left(\pi^{*}\left(\left[\theta_{i}\right]\right)\right)=\pi^{*}\left[\theta_{i}\right]
$$

for all $g \in G$. Therefore, we obtain from (4-3) and (4-4)

$$
\begin{aligned}
\left\langle\Theta_{i}, \tilde{c}_{j}\right\rangle & =\frac{1}{|G|}\left\langle\sum_{g \in G / H} \delta(g) g^{*}\left(\pi^{*}\left(\left[\theta_{i}\right]\right)\right),\left[\sum_{h \in H} h_{\#}\left(\widetilde{c}_{j}\right)\right]\right\rangle \\
& =\frac{|G / H|}{|G|}\left\langle\pi^{*}\left(\left[\theta_{i}\right]\right),\left[\sum_{h \in H} h_{\#}\left(\tilde{c}_{j}\right)\right]\right\rangle \\
& =\frac{|G / H|}{|G|}\left\langle\left[\theta_{i}\right], \pi_{*}\left[\sum_{h \in H} h_{\#}\left(\tilde{c}_{j}\right)\right]\right\rangle=\frac{|G / H|}{|G|}\left\langle\left[\theta_{i}\right],|H|\left[c_{j}\right]\right\rangle,
\end{aligned}
$$


where the last equality holds by (4-2). It follows that

$$
\left\langle\Theta_{i}, \tilde{c}_{j}\right\rangle=\left\langle\left[\theta_{i}\right],\left[c_{j}\right]\right\rangle=\delta_{i j},
$$

as claimed.

To prove (4), we observe that a similar computation as in the proof of (3) shows that

$$
\left\langle\Theta_{i}, c\right\rangle=\left\langle\left[\theta_{i}\right],\left[\pi_{\#}(c)\right]\right\rangle .
$$

The claim (4) follows because $\left[\pi_{\#}(c)\right] \in H_{1}(X / H, \mathbb{Z})$ can be written as a linear combination of the basis $\left[c_{1}\right], \ldots,\left[c_{n}\right]$ with integer coefficients and because $\left\langle\left[\theta_{i}\right],\left[c_{j}\right]\right\rangle=\delta_{i j}$ by the choice of the bases. This concludes the proof of Proposition 4.1.

\section{The Abel-Jacobi map}

Still using Notation 2.8, we construct in this section a map $\Psi: X \rightarrow \mathbb{R}^{n} / \mathbb{Z}^{n}$ and study its equivariance properties with respect to the action of $G$ on $X$. The construction of the map $\Psi$ is motivated by the construction of the classical Abel-Jacobi map of a Riemann surface into its Jacobian.

To begin with we consider the linear map

$$
\tilde{\Psi}: C_{1}(X ; \mathbb{R}) \rightarrow \mathbb{R}^{n}, \quad \tilde{\Psi}(c)=\left(\left\langle\Theta_{i}, c\right\rangle\right)_{i=1, \ldots, n},
$$

given by evaluating the 1 -cocycles $\Theta_{1}, \ldots, \Theta_{n}$ in Proposition 4.1 on singular chains. By (4) in Proposition 4.1, the image of $Z_{1}(X ; \mathbb{Z})$ under $\widetilde{\Psi}$ is contained in $\mathbb{Z}^{n}$. In particular, $\widetilde{\Psi}$ induces a map

$$
\Psi^{\prime}: C_{1}(X ; \mathbb{R}) / Z_{1}(X ; \mathbb{Z}) \rightarrow \mathbb{R}^{n} / \mathbb{Z}^{n}
$$

In order to obtain a map defined on $X$ we choose a base point $x_{0}$. Suppose that $x \in X$ is another point and that $I$ and $I^{\prime}$ are two paths starting at $x_{0}$ and ending in $x$. Considering both paths as 1 -chains we have $\Psi^{\prime}(I)=\Psi^{\prime}\left(I^{\prime}\right)$ because $I-I^{\prime}$ is a 1-cycle. It follows that the value $\Psi^{\prime}(I)$ does not depend on the chosen path $I$ but only on the point $x$. We obtain hence a well-defined map

$$
\Psi: X \rightarrow \mathbb{R}^{n} / \mathbb{Z}^{n}
$$

We will refer to $\Psi$ as the Abel-Jacobi map.

Proposition 5.1 The Abel-Jacobi map $\Psi: X \rightarrow \mathbb{R}^{n} / \mathbb{Z}^{n}$ descends to a homotopy equivalence $\widehat{\Psi}: \mathbb{T}^{n}=X / H \rightarrow \mathbb{R}^{n} / \mathbb{Z}^{n}$. 
We start by investigating how the action of $G$ on $X$ is reflected by the Abel-Jacobi map. In order to do so we consider $\mathbb{R}^{n} / \mathbb{Z}^{n}$ as an abelian group.

Lemma 5.2 For all $g \in G$ there is $A_{g} \in \mathbb{R}^{n} / \mathbb{Z}^{n}$ with

$$
\Psi(g(x))=\delta(g) \Psi(x)+A_{g}
$$

for all $x \in X$.

Proof For $x \in X$ denote by $I_{x}$ a path from $x_{0}$ to $x$. In order to compute $\Psi(g(x))$ we consider the juxtaposition $I_{g\left(x_{0}\right)}+g\left(I_{x}\right)$ of $I_{g\left(x_{0}\right)}$ and $g\left(I_{x}\right)$. We have thus $\Psi(g(x))=\Psi^{\prime}\left(I_{g\left(x_{0}\right)}+g\left(I_{x}\right)\right)=\Psi^{\prime}\left(I_{g\left(x_{0}\right)}\right)+\Psi^{\prime}\left(g\left(I_{x}\right)\right)=\Psi\left(g\left(x_{0}\right)\right)+\Psi^{\prime}\left(g\left(I_{x}\right)\right)$.

We set $A_{g}=\Psi\left(g\left(x_{0}\right)\right)$ and compute

$$
\Psi^{\prime}\left(g\left(I_{x}\right)\right)=\left(\left\langle\Theta_{i}, g I_{x}\right\rangle\right)_{i=1, \ldots, n} .
$$

We have for $i=1, \ldots, n$

$$
\left\langle\Theta_{i}, g\left(I_{x}\right)\right\rangle=\left\langle\Theta_{i}, g_{\#}\left(I_{x}\right)\right\rangle=\left\langle g^{\#} \Theta_{i}, I_{x}\right\rangle=\delta(g)\left\langle\Theta_{i}, I_{x}\right\rangle,
$$

where the last equation holds by Proposition 4.1. The claim follows.

Clearly, the map

$$
G \times \mathbb{R}^{n} / \mathbb{Z}^{n} \rightarrow \mathbb{R}^{n} / \mathbb{Z}^{n}, \quad(g, x) \mapsto \delta(g) x+A_{g},
$$

is an action of $G$ on $\mathbb{R}^{n} / \mathbb{Z}^{n}$.

Lemma 5.3 The restriction of the $G$-action (5-1) to $H$ is trivial.

Proof Notice that for any $h \in H$ the path $I_{h\left(x_{0}\right)}$ projects to a closed loop in $X / H$. In other words, $\pi_{\#}\left(I_{h\left(x_{0}\right)}\right)$ is a 1 -cycle in $X / H=\mathbb{T}^{n}$. It follows from (4) in Proposition 4.1 that $\tilde{\Psi}\left(I_{h\left(x_{0}\right)}\right) \in \mathbb{Z}^{n}$, meaning that

$$
A_{h}=\tilde{\Psi}\left(I_{h\left(x_{0}\right)}\right) \bmod \mathbb{Z}^{n}=0 .
$$

Since $H \subset \operatorname{Ker}(\delta)$ we deduce from Lemma 5.2 that the action of $H$ on $\mathbb{R}^{n} / \mathbb{Z}^{n}$ is trivial.

Proof of Proposition 5.1 Lemma 5.3 implies directly that the Abel-Jacobi map $\Psi$ descends to a map

$$
\widehat{\Psi}: X / H \rightarrow \mathbb{R}^{n} / \mathbb{Z}^{n}
$$


We claim now that $\hat{\Psi}$ is a homotopy equivalence. In order to see that this is the case let $c_{1}$ be a loop in $X / H=\mathbb{T}^{n}$ representing the homology class $\left[c_{1}\right] \in H_{1}(X / H ; \mathbb{Z})=$ $\pi_{1}(X / H)$ chosen in the previous section. Suppose also that $c_{1}$ is based at $\pi\left(x_{0}\right)$ and avoids $Z_{f}$, the preimage of the branching locus of $f$. This last condition implies that there is a unique lift $\tilde{c}_{1}$ of $c_{1}$ starting at $x_{0}$. Applying Proposition 4.1(3) to $\tilde{c}_{1}$ we obtain that

$$
\widetilde{\Psi}\left(\widetilde{c}_{1}\right)=(1,0, \ldots, 0) .
$$

Identifying $\pi_{1}\left(\mathbb{R}^{n} / \mathbb{Z}^{n}\right)$ with the deck-transformation group $\mathbb{Z}^{n}$ of the cover $\mathbb{R}^{n} \rightarrow$ $\mathbb{R}^{n} / \mathbb{Z}^{n}$ we have that the image $\hat{\Psi}\left(c_{1}\right)$ of $c_{1}$ is homotopic to the first standard generator of $\pi_{1}\left(\mathbb{R}^{n} / \mathbb{Z}^{n}\right) \simeq \mathbb{Z}^{n}$. Arguing in the same way for $c_{2}, \ldots, c_{n}$ we have proved that the homomorphism

$$
\pi_{1}(\widehat{\Psi}): \pi_{1}\left(X / H, \pi\left(x_{0}\right)\right) \rightarrow \pi_{1}\left(\mathbb{R}^{n} / \mathbb{Z}^{n}, \widehat{\Phi}\left(\pi\left(x_{0}\right)\right)\right)
$$

induced by $\hat{\Psi}$ maps the basis $\left[c_{1}\right], \ldots,\left[c_{n}\right]$ to the standard basis of $\mathbb{Z}^{n}=\pi_{1}\left(\mathbb{R}^{n} / \mathbb{Z}^{n}\right)$. In other words, $\pi_{1}(\hat{\Psi})$ is an isomorphism. Since both $X / H$ and $\mathbb{R}^{n} / \mathbb{Z}^{n}$ are tori, and hence have contractible universal coverings, this implies that $\Psi: X / H \rightarrow \mathbb{R}^{n} / \mathbb{Z}^{n}$ is a homotopy equivalence, as we wanted to show.

We can now start drawing a diagram to which we will continue adding arrows and objects in the next section:

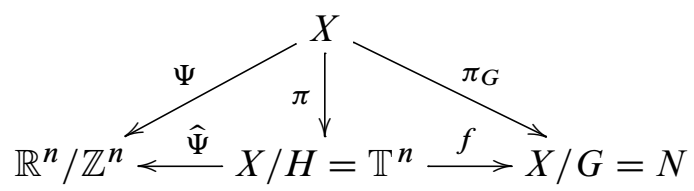

\section{The subgroup $K \subset G$}

Still using Notation 2.8 define

$$
K=\operatorname{Ker}(\delta)
$$

to be the kernel of the homomorphism $\delta$; see (3-2). By construction $K$ is a normal subgroup of $G$ with at most index 2. Notice that $H \subset K=\operatorname{Ker}(\delta)$ and that it follows from Corollary 3.4 that $K=G$ if the dimension $n$ of the involved manifolds is odd.

Remark In the next section, we will also prove that $K=G$ if $n$ is even.

By Lemma 5.2, the action (5-1) of $G$ on $\mathbb{R}^{n} / \mathbb{Z}^{n}$ restricts to an action of $K$ by translations. Observe that since $H \subset K$, this action is not effective by Lemma 5.3. In spite of this, we will denote by $\left(\mathbb{R}^{n} / \mathbb{Z}^{n}\right) / K$ the quotient of $\mathbb{R}^{n} / \mathbb{Z}^{n}$ under the action by translations of $K$. In this section we prove: 
Proposition 6.1 The homotopy equivalence $\hat{\Psi}: X / H \rightarrow \mathbb{R}^{n} / \mathbb{Z}^{n}$ descends to a map $\bar{\Psi}: X / K \rightarrow\left(\mathbb{R}^{n} / \mathbb{Z}^{n}\right) / K$. In particular, the following holds:

(1) The orbit map $p_{1}: \mathbb{R}^{n} / \mathbb{Z}^{n} \rightarrow\left(\mathbb{R}^{n} / \mathbb{Z}^{n}\right) / K$ has degree $|K / H|$.

(2) $H$ is a normal subgroup of $K$.

(3) The action of $K / H$ on $\mathbb{R}^{n} / \mathbb{Z}^{n}$ is effective and $\left(\mathbb{R}^{n} / \mathbb{Z}^{n}\right) / K$ is a torus.

(4) The action of $K / H$ on $X / H$ is free. In particular, the space $X / K$ is in fact a manifold and the orbit map $p_{2}: X / H \rightarrow X / K$ is a covering map.

(5) The map $\bar{\Psi}: X / K \rightarrow\left(\mathbb{R}^{n} / \mathbb{Z}^{n}\right) / K$ is a homotopy equivalence.

Proof The fact that $\hat{\Psi}: X / H \rightarrow \mathbb{R}^{n} / \mathbb{Z}^{n}$ descends to a map $\bar{\Psi}: X / K \rightarrow\left(\mathbb{R}^{n} / \mathbb{Z}^{n}\right) / K$ follows just from the definitions and is left to the reader. Notice that since $K$ acts on the $n$-dimensional torus $\mathbb{R}^{n} / \mathbb{Z}^{n}$ by translations, the quotient is an $n$-dimensional a torus as well. This proves the second part of (3).

At this point we can enlarge the diagram above as follows:

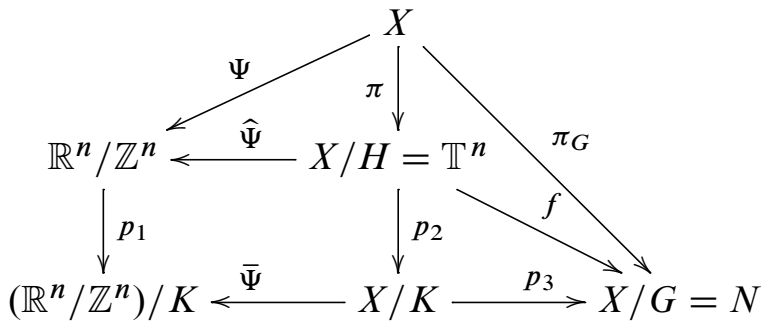

where $p_{1}, p_{2}$ and $p_{3}$ are the obvious orbit maps.

Since the square in the left bottom corner of the diagram commutes we have that

$$
\operatorname{deg}\left(p_{1}\right) \operatorname{deg}(\widehat{\Psi})=\operatorname{deg}\left(p_{1} \circ \hat{\Psi}\right)=\operatorname{deg}\left(\bar{\Psi} \circ p_{2}\right)=\operatorname{deg}(\bar{\Psi}) \operatorname{deg}\left(p_{2}\right) .
$$

Taking into account that $\widehat{\Psi}$ is a homotopy equivalence and that $p_{2}$ has degree $|K / H|$ we obtain

$$
\operatorname{deg}\left(p_{1}\right)=|K / H| \operatorname{deg}(\bar{\Psi}) .
$$

On the other hand, $p_{1}$ has positive degree. This implies that $\bar{\Psi}$ has to have positive degree as well; hence

$$
\operatorname{deg}\left(p_{1}\right) \geq|K / H|
$$

On the other hand, $\operatorname{deg}\left(p_{1}\right) \leq|K / H|$ because the subgroup $H$ of $K$ acts trivially on $\mathbb{R}^{n} / \mathbb{Z}^{n}$. We have proved (1).

Recall that $H$ is contained in the kernel of the homomorphism

$$
K \rightarrow \operatorname{Aut}\left(\mathbb{R}^{n} / \mathbb{Z}^{n}\right)
$$


by Lemma 5.3. The image of this homomorphism has $\operatorname{deg}\left(p_{1}\right)=|K / H|$ elements. It follows that $H$ is in fact precisely the kernel of this homomorphism and that it is therefore normal in $K$. We have proved (2) and the first part of (3).

Now $H$ is normal in $K$, so $K / H$ acts on $X / H$ and that $X / K=(X / H) /(K / H)$. We claim that the action of $K / H$ on $X / H$ is free. In order to see this, suppose that we have $x \in X / H$ and $k \in K / H$ with $k x=x$. Then we have

$$
\widehat{\Psi}(x)=\widehat{\Psi}(k x)=k \widehat{\Psi}(x) .
$$

Since the action of $K / H$ on $\mathbb{R}^{n} / \mathbb{Z}^{n}$ is effective and by translations, an element $k \in K / H$ can only fix the point $\Psi(x) \in \mathbb{R}^{n} / \mathbb{Z}^{n}$ if $k$ is the neutral element in $K / H$. We have proved (4).

It remains to show that the map $\bar{\Psi}: X / K \rightarrow\left(\mathbb{R}^{n} / \mathbb{Z}^{n}\right) / K$ is a homotopy equivalence. As in the proof of Proposition 5.1, it suffices to show that $\left(\pi_{1}\right)_{*}(\bar{\Psi}): \pi_{1}(X / K) \rightarrow$ $\pi_{1}\left(\left(\mathbb{R}^{n} / \mathbb{Z}^{n}\right) / K\right)$ is an isomorphism. Notice at this point that we have the following commutative diagram:

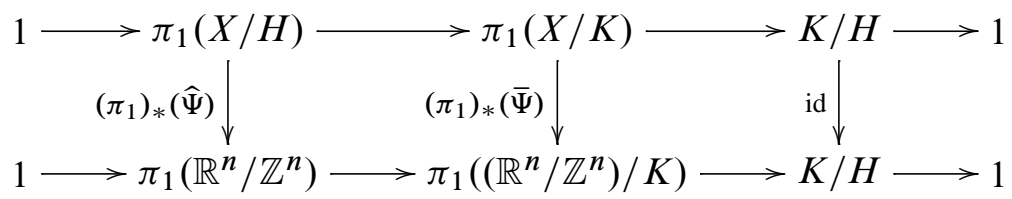

Since the first and third vertical arrows are isomorphisms it follows that the middle arrow is an isomorphism as well. This concludes the proof of Proposition 6.1.

We conclude this section with some remarks needed to prove Theorem 1.2 below. The following is just a direct consequence of parts (3) and (5) of Proposition 6.1:

Corollary 6.2 The manifold $X / K$ is homotopy equivalent to an $n$-torus.

Recall now that $K \subset G$ is normal of at most index 2 because it is the kernel of the homomorphism (3-2). In particular, the group $G / K$ acts on $X / K$ with $X / G=$ $(X / K) /(G / K)$ and the map $p_{3}: X / K \rightarrow X / G=N$ in (6-1) is just the orbit map. We prove now:

Lemma 6.3 If $K \neq G$ denote by $\sigma$ the nontrivial element in $G / K$. Then $\sigma$ acts as $-\mathrm{id}$ on $H^{1}(X / K ; \mathbb{R})$.

We suggest the reader to compare with Corollary 3.5. 
Proof Denote by $\tilde{\sigma} \in G$ a representative of $\sigma$ in $G$. Notice that, by the very definition of the action (5-1), $\widetilde{\sigma}$ acts on $H^{1}\left(\mathbb{R}^{n} / \mathbb{Z}^{n} ; \mathbb{R}\right)$ by -id. Since $K$ acts on $\mathbb{R}^{n} / \mathbb{Z}^{n}$ by translations, it follows that $\sigma$ also acts on $H^{1}\left(\left(\mathbb{R}^{n} / \mathbb{Z}^{n}\right) / K ; \mathbb{R}\right)$ by - id. Since the homotopy equivalence $\bar{\Psi}$ satisfies $\bar{\Psi}(\sigma(x))=\sigma(\bar{\Psi}(x))$, we deduce that $\sigma$ acts on $H^{1}(X / K ; \mathbb{R})$ as - id as well.

\section{The final step of the proof of Theorem 1.2}

In this section we conclude the proof of Theorem 1.2; as always, we use Notation 2.8. We copy (6-1) here for the convenience of the reader:

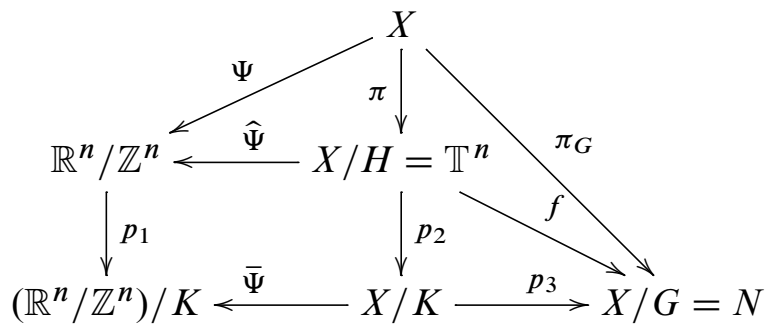

Having this diagram in mind, we conclude the proof of Theorem 1.2:

Theorem 1.2 Let $N$ be a closed, connected, and oriented $n$-manifold, $n \geq 2$ so that $\operatorname{dim} H^{r}(N ; \mathbb{R})=\operatorname{dim} H^{r}\left(\mathbb{T}^{n} ; \mathbb{R}\right)$ for some $1 \leq r<n$. Then every branched cover $f: \mathbb{T}^{n} \rightarrow N$ is a cover. In particular, every $\pi_{1}$-surjective branched cover $\mathbb{T}^{n} \rightarrow N$ is a homeomorphism.

Proof If $G / K$ is the trivial group then $p_{3}=\mathrm{id}$ and, in particular, we have

$$
f=p_{3} \circ p_{2}=p_{2} \text {. }
$$

By Proposition 6.1, the map $p_{2}$, and hence $f$, is a covering map. Thus it suffices to show that $K=G$.

Seeking a contradiction assume that $K \neq G$. Hence $G / K$ is the group of order two. As in Lemma 6.3, denote by $\sigma$ the nontrivial element in $G / K$. It follows from Corollary 6.2 that $X / K$ is homotopy equivalent to an $n$-torus. Furthermore, it follows from Lemma 6.3 that $\sigma$ acts on $H^{S}(X / K ; \mathbb{R})$ by multiplication by $(-1)^{S}$. Since $(X / K) /(G / K)=X / G=N$ is an orientable manifold, this contradicts Corollary 2.7. The proof is complete. 


\section{Brief discussion of Theorem 1.1}

Theorem 1.1 There is no branched cover from the 4-dimensional torus $\mathbb{T}^{4}$ to $\#^{3}\left(\mathbb{S}^{2} \times \mathbb{S}^{2}\right)$, the connected sum of three copies of $\mathbb{S}^{2} \times \mathbb{S}^{2}$. On the other hand, there are, a fortiori $\pi_{1}$-surjective, maps $\mathbb{T}^{4} \rightarrow \#^{3}\left(\mathbb{S}^{2} \times \mathbb{S}^{2}\right)$ of arbitrarily large degree.

The claim that there is no branched covering $\mathbb{T}^{4} \rightarrow \#^{3}\left(\mathbb{S}^{2} \times \mathbb{S}^{2}\right)$ follows directly from Theorem 1.2. Maps of arbitrarily large degree

$$
f: \mathbb{T}^{4} \rightarrow \#^{3}\left(\mathbb{S}^{2} \times \mathbb{S}^{2}\right)
$$

can either be constructed directly, or be shown to exist using the work of Duan and Wang [10]. These authors prove namely the following remarkable theorem:

Theorem (Duan-Wang [10, Theorem 3]) Suppose $M$ and $L$ are closed oriented 4-manifolds and denote by $\bar{H}^{2}(M ; \mathbb{Z})$ and $\bar{H}^{2}(L ; \mathbb{Z})$ the torsion free part of the corresponding second cohomology group. Let also $\alpha^{*}$ and $\beta^{*}$ be respectively bases of $\bar{H}^{2}(M ; \mathbb{Z})$ and $\bar{H}^{2}(L ; \mathbb{Z})$ and let $A$ and $B$ be the intersection matrices on $M$ and $L$ with respect to the bases $\alpha^{*}$ and $\beta^{*}$. Finally, let $P$ be an $m-b y-l-m a t r i x$, where $m=\operatorname{dim} \bar{H}^{2}(M)$ and $l=\operatorname{dim} \bar{H}^{2}(L)$.

If $L$ is simply connected, then there is a map $f: M \rightarrow L$ of degree $k$ such that $f^{*}\left(\beta^{*}\right)=\alpha^{*} P$ if and only if $P^{t} A P=k B$. Moreover, there is a map $f: M \rightarrow L$ of degree 1 if and only if the intersection form of $L$ is isomorphic to a direct summand of the intersection form of $M$.

In [10, Example 4], this is applied in the particular case of maps $\mathbb{T}^{4} \rightarrow \#^{3}\left(\mathbb{S}^{2} \times \mathbb{S}^{2}\right)$ to obtain mappings of every degree. We finish by recalling this example. Let $\left[\theta_{1}\right], \ldots,\left[\theta_{4}\right]$ be generators of $H^{1}\left(\mathbb{T}^{4} ; \mathbb{Z}\right)$ as in Section 4 . The basis $\left(\left[\theta_{i}\right] \wedge\left[\theta_{j}\right]\right)_{1 \leq i<j \leq 4}$ of $H^{2}\left(\mathbb{T}^{4} ; \mathbb{Z}\right)$ can be ordered so that the intersection form of $H^{2}\left(\mathbb{T}^{4} ; Z\right)$ reads as

$$
\oplus^{3}\left(\begin{array}{ll}
0 & 1 \\
1 & 0
\end{array}\right) \text {. }
$$

Similarly, we can fix 2 -spheres in $S_{1}, \ldots, S_{6}$ in $\#^{3}\left(\mathbb{S}^{2} \times \mathbb{S}^{2}\right)$, so that $\left[S_{1}\right], \ldots,\left[S_{6}\right]$ is a basis of $H_{2}\left(\#^{3}\left(\mathbb{S}^{2} \times \mathbb{S}^{2}\right) ; \mathbb{Z}\right)$ and that the intersection form of $H^{2}\left(\#^{3}\left(\mathbb{S}^{2} \times \mathbb{S}^{2}\right) ; \mathbb{R}\right)$ in the corresponding dual basis reads as

$$
\bigoplus^{3}\left(\begin{array}{ll}
0 & 1 \\
1 & 0
\end{array}\right)
$$

Thus the linear mapping given by the matrix

$$
P=\bigoplus^{3}\left(\begin{array}{ll}
0 & k \\
1 & 0
\end{array}\right)
$$

with respect to these bases of $H^{2}\left(\#^{3}\left(\mathbb{S}^{2} \times \mathbb{S}^{2}\right) ; \mathbb{R}\right)$ and $H^{2}\left(\mathbb{T}^{4} ; \mathbb{R}\right)$ yields the result. 


\section{References}

[1] J W Alexander, Note on Riemann spaces, Bull. Amer. Math. Soc. 26 (1920) 370-372 MR1560318

[2] I Berstein, A L Edmonds, The degree and branch set of a branched covering, Invent. Math. 45 (1978) 213-220 MR0474261

[3] I Berstein, A L Edmonds, On the construction of branched coverings of lowdimensional manifolds, Trans. Amer. Math. Soc. 247 (1979) 87-124 MR517687

[4] M Bonk, J Heinonen, Quasiregular mappings and cohomology, Acta Math. 186 (2001) 219-238 MR1846030

[5] G E Bredon, Orientation in generalized manifolds and applications to the theory of transformation groups, Michigan Math. J. 7 (1960) 35-64 MR0116342

[6] G E Bredon, Introduction to compact transformation groups, Pure and Applied Math. 46, Academic Press, New York (1972) MR0413144

[7] G E Bredon, Topology and geometry, Graduate Texts in Math. 139, Springer, New York (1997) MR1700700

[8] A V Černavskiü, Finite-to-one open mappings of manifolds, Mat. Sb. 65 (107) (1964) 357-369 MR0172256 In Russian; translated in Amer. Math. Soc., Translat., II. Ser. 100 (1972) 253-267

[9] F Connolly, J F Davis, Q Khan, Topological rigidity and $H_{1}$-negative involutions on tori arXiv:1102.2660

[10] H B Duan, S C Wang, Non-zero degree maps between $2 n$-manifolds, Acta Math. Sin. (Engl. Ser.) 20 (2004) 1-14 MR2056551

[11] A L Edmonds, Deformation of maps to branched coverings in dimension three, Math. Ann. 245 (1979) 273-279 MR553345

[12] M Gromov, Hyperbolic manifolds, groups and actions, from "Riemann surfaces and related topics: Proceedings of the 1978 Stony Brook Conference (State Univ. New York, 1978)" (I Kra, B Maskit, editors), Ann. of Math. Stud. 97, Princeton Univ. Press (1981) 183-213 MR624814

[13] M Gromov, Metric structures for Riemannian and non-Riemannian spaces, Progress in Math. 152, Birkhäuser, Boston, MA (1999) MR1699320 Based on the 1981 French original [MR0682063], With appendices by M Katz, P Pansu and S Semmes, Translated from the French by S M Bates

[14] A Hatcher, Algebraic topology, Cambridge Univ. Press (2002) MR1867354

[15] H M Hilden, Three-fold branched coverings of $S^{3}$, Amer. J. Math. 98 (1976) 989-997 MR0425968

[16] U Hirsch, Über offene Abbildungen auf die 3-Sphäre, Math. Z. 140 (1974) 203-230 MR0362313 
[17] J F P Hudson, Piecewise linear topology, Univ. of Chicago Lecture Notes, Benjamin, New York-Amsterdam (1969) MR0248844 Prepared with the assistance of J L Shaneson and $\mathrm{J}$ Lees

[18] M Iori, R Piergallini, 4-Manifolds as covers of the 4-sphere branched over nonsingular surfaces, Geom. Topol. 6 (2002) 393-401 MR1914574

[19] J M Montesinos, Three-manifolds as 3-fold branched covers of $S^{3}$, Quart. J. Math. Oxford Ser. 27 (1976) 85-94 MR0394630

[20] R Piergallini, Four-manifolds as 4-fold branched covers of $S^{4}$, Topology 34 (1995) 497-508 MR1341805

[21] S Rickman, Existence of quasiregular mappings, from "Holomorphic functions and moduli, Vol. I (Berkeley, CA, 1986)" (D Drasin, C J Earle, F W Gehring, I Kra, A Marden, editors), Math. Sci. Res. Inst. Publ. 10, Springer, New York (1988) 179-185 MR955819

[22] S Rickman, Quasiregular mappings, Ergeb. Math. Grenzgeb. 26, Springer, Berlin (1993) MR1238941

[23] S Rickman, Simply connected quasiregularly elliptic 4-manifolds, Ann. Acad. Sci. Fenn. Math. 31 (2006) 97-110 MR2210111

[24] C P Rourke, B J Sanderson, Introduction to piecewise-linear topology, Springer Study Edition, Springer, Berlin (1982) MR665919

[25] J Väisälä, Discrete open mappings on manifolds, Ann. Acad. Sci. Fenn. Ser. A I No. 392 (1966) 10 MR0200928

Department of Mathematics and Statistics, University of Helsinki

PO Box 68 (Gustaf Hällströmin katu 2b), FI-00014 Helsinki, Finland

Mathematics Department, University of British Columbia

1984 Mathematics Road, Vancouver BC V6T 1Z2, Canada

pekka.pankka@helsinki.fi, jsouto@math.ubc.ca

http://www.helsinki.fi/ pankka, http://www.math.ubc.ca/ jsouto

Proposed: David Gabai

Seconded: Benson Farb, Ronald Fintushel
Received: 25 January 2011

Revised: 27 January 2012 


\title{
GEOMETRY \& TOPOLOGY
}

\author{
www.msp.warwick.ac.uk/gt
}

EDITORS

MANAGING EDITORS

Colin Rourke

gt@maths.warwick.ac.uk

Mathematics Institute

University of Warwick

Coventry CV4 7AL UK

\author{
Brian Sanderson \\ gt@maths.warwick.ac.uk \\ Mathematics Institute \\ University of Warwick \\ Coventry CV4 7AL \\ UK
}

\section{BOARD OF EDITORS}

\author{
Joan Birman Columbia University \\ jb@math.columbia.edu
}

Martin Bridson Imperial College, London m.bridson@ic.ac.uk

Jim Bryan University of British Columbia jbryan@math.ubc.ca

Ben Chow University of California, San Diego benchow@math.ucsd.edu

Ralph Cohen Stanford University ralph@math.stanford.edu

Tobias Colding Massachusetts Institute of Technology colding@math.mit.edu

Simon Donaldson Imperial College, London s.donaldson@ic.ac.uk

Bill Dwyer University of Notre Dame dwyer.1@nd.edu

Yasha Eliashberg Stanford University eliash@math.stanford.edu

Benson Farb University of Chicago farb@math.uchicago.edu

Steve Ferry Rutgers University sferry@math.rutgers.edu

Ron Fintushel Michigan State University ronfint@math.msu.edu

Mike Freedman Microsoft Research michaelf@microsoft.com

David Gabai Princeton University gabai@princeton.edu

Paul Goerss Northwestern University pgoerss@math.northwestern.edu

Lothar Göttsche Abdus Salam Int. Centre for Th. Physics gottsche@ictp.trieste.it

Tom Goodwillie Brown University tomg@math.brown.edu
Cameron Gordon

Eleny Ionel

Vaughan Jones

Robion Kirby

Frances Kirwan

Wolfgang Lück

Haynes Miller

Shigeyuki Morita

John Morgan

Tom Mrowka

Walter Neumann

Jean-Pierre Otal

Peter Ozsvath

Leonid Polterovich

Colin Rourke

Ron Stern

Peter Teichner

\author{
Walter Neumann \\ gt@maths.warwick.ac.uk \\ Department of Mathematics \\ Barnard College, Columbia University \\ New York, NY 10027-6902 \\ USA
}

University of Texas

gordon@math.utexas.edu

Stanford University

ionel@math.stanford.edu

University of California, Berkeley vfr@math.berkeley.edu

University of California, Berkeley kirby@math.berkeley.edu

University of Oxford

frances.kirwan@balliol.oxford.ac.uk

Universität Münster

lueck@math.uni-muenster.de

Massachusetts Institute of Technology hrm@math.mit.edu

University of Tokyo morita@ms.u-tokyo.ac.jp

Columbia University jm@math.columbia.edu

Massachusetts Institute of Technology mrowka@math.mit.edu

Columbia University neumann@math.columbia.edu

Université d'Orleans jean-pierre.otal@univ-orleans.fr

Columbia University ozsvath@math.columbia.edu

Tel Aviv University polterov@post.tau.ac.il

University of Warwick cpr@maths.warwick.ac.uk

University of California, Irvine rstern@math.uci.edu

University of California, Berkeley teichner@math.berkeley.edu

\section{Gang Tian Massachusetts Institute of Technology}

tian@math.mit.edu

See inside back cover or www.msp.warwick.ac.uk/gt for submission instructions.

The subscription price for 2012 is US \$375/year for the electronic version, and \$575/year ( $+\$ 60$ shipping outside the US) for print and electronic. Subscriptions, requests for back issues from the last three years and changes of subscribers address should be sent to Mathematical Sciences Publishers, Department of Mathematics, University of California, Berkeley, CA 94704-3840, USA. Geometry \& Topology is indexed by Mathematical Reviews, Zentralblatt MATH, Current Mathematical Publications and the Science Citation Index.

Geometric \& Topology (ISSN 1364-0380 electronic, 1465-3060 printed) at Mathematical Sciences Publisher, Department of Mathematics, University of California, Berkeley, CA 94720-3840 is published continuously online. Periodical rate postage paid at Berkeley, CA 94704, and additional mailing offices.

G\&T peer review and production are managed by EditFLOW ${ }^{\mathrm{TM}}$ from Mathematical Sciences Publishers. 


\section{GEOMETRY \& TOPOLOGY}

Volume 16 Issue 3 (pages 1247-1880) 2012

Deformation spaces of Kleinian surface groups are not locally connected

\section{AARON D MAGID}

On the nonexistence of certain branched covers

PEKKA PANKKA and JUAN SOUTO

Geodesic flow for CAT(0)-groups

ARTHUR BARTELS and WOLFGANG LÜCK

All finite groups are involved in the mapping class group

Gregor MASBAUM and Alan W REID

Noncollapsing in mean-convex mean curvature flow

BEN ANDREWS

Whitney tower concordance of classical links

JAMES CONANT, RoB SCHNEIDERMAN and PETER

TEICHNER

Blob homology

SCOTT MORRISON and KEVIN WALKER

Prym varieties of spectral covers

TAMÁs HAUSEL and CHRISTIAN PAULY

Legendrian and transverse cables of positive torus knots

John B ETNYRE, Douglas J LAFOUNTAIN and BÜLENT

TOSUN

Obstructions to stably fibering manifolds

WOLFGANG STEIMLE

Towards representation stability for the second homology of the

Torelli group

Søren K Boldsen and Mia Hauge Dollerup

$\mathrm{SO}(3)$-Donaldson invariants of $\mathbb{C P}^{2}$ and mock theta functions

ANDREAS MALMENDIER and KEN ONO

Rigidity of certain solvable actions on the sphere

MASAYUKI ASAOKA

The Novikov conjecture and geometry of Banach spaces 